\title{
Discriminated avoidance learning and reversal by goldfish in a shuttlebox using a linear presentation procedure
}

\author{
DOMINIC J. ZERBOLIO, JR. \\ University of Missouri, St. Louis, Missouri 63121
}

\begin{abstract}
Goldfish, trained in a shuttlebox with cues and shock controlled by a linear presentation procedure, learned to control prevailing cue states and shock, and thus to discriminatively avoid shock presentation. The linear presentation procedure, adapted for the shuttlebox from the sequence of cue presentation occurring in the Y-maze, utilized three cues on each trial, a trial-start (TS) cue, a shock-paired (S-) cue, and an unpaired (S+) cue. At trial onset (TS cue), the goldfish had $10 \mathrm{sec}$ to respond. The first response produced, via response-contingent programming, either the shock-paired $(\mathrm{S}-$ ) or unpaired (S+) cue, and subsequent responses produced alternation of $\mathrm{S}+$ and S-. The shock was omitted only if the S+ cue state prevailed $10 \mathrm{sec}$ after trial onset. All other cue states (TS and S-) were paired with shock. Goldfish learned to respond and control the prevailing cue state so that the S+ cue prevailed at $10 \mathrm{sec}$ posttrial onset for a variety of different color-cue combinations and also learned to reverse their originally learned cue preference when the color cues were reversed. The linear presentation procedure represents an alternative discrimination learning procedure that appears to be free of the interpretational problems encountered in training goldfish in the shuttlebox apparatus with other one- and two-stimulus procedures.
\end{abstract}

Can goldfish learn a discriminated avoidance response in the shuttlebox? The question has stirred a bit of controversy. To understand the question and the problem posed by the goldfish's behavior, one must understand what an avoidance response is in the shuttlebox and, more importantly, how it is measured.

The typical discriminated avoidance procedure in the shuttlebox involves presenting a signal cue for a timed period and programming shock to occur at the end of the timed period unless the animal meets a specific response requirement. If the animal meets the response requirement in the timed period, the shock is omitted. Because shock omission is contingent upon the animal's response, the procedure is, by definition, an instrumental procedure. If the animal "learns" to avoid, it will respond in an increasing proportion of the timed periods. The learning measure is the incidence (i.e., the probability of responding) in the timed periods. It is tacitly assumed that the animal will not respond in an increasing proportion of timed periods unless it is compelled to do so by shock. Another way of saying this is to say that the animal is expected to habituate responding (i.e., not shuttle) in procedures that do not involve shock. It is only when the "habituated" response and the "learned" response can be clearly separated by the learning measure that an unequivocal learning interpretation can be given. With these distinctions and assumptions in mind, we can proceed to the results of running goldfish in the shuttlebox and to the implications of previous studies.

The discriminated avoidance work employing goldfish in the shuttlebox can be conveniently divided into three procedural types, which are distinguished by the number of stimuli used as signals and by the type of response required. Specifically, the three types are the active one-signal procedure, the passive or punishment one-signal procedure, and the successive presentation two-signal procedure. Keeping in mind that the response measure is the incidence of the response in the timed period prior to the programmed shock, the data resulting from the active one-signal procedures show goldfish "learning" (responding in increasing proportions of the timed periods) when the timed period is paired with shock instrumentally (response-contingent shock omission), when the timed and signaled period is paired with shock classically (shock occurs on every trial), when the shock is eliminated entirely (shock turned off), and when shock and the signal are eliminated (both turned off) (Steiner, 1971; Woodard \& Bitterman, 1973; Zerbolio \& Wickstra, 1978a, 1978b). Since goldfish show increased responding either with or without shock, and with or without the signal differentiating the timed period (trial) from the ITI, it seems questionable that the response increase represents any sort of learning. If one accepts this conclusion, then issues such as the shock pairing 
(classical or instrumental) or response-contingent signal termination become secondary.

Steiner (1971) clearly anticipated this result. When Steiner found that goldfish not only do not habituate the shuttle response, but in fact increase the proportion of timed and signaled periods with response with the shock eliminated, she concluded that the response increase measure might be either inadequate or meaningless as a measure of learning. Obviously, the assumption that the animal in the shuttlebox will habituate, or stop its shuttling response, unless it is compelled to do otherwise by shock, is not valid for the goldfish. But, since goldfish do not habituate in the shuttlebox, perhaps they can be taught to discriminatively inhibit their shuttling response.

The procedure to train an animal to discriminately inhibit responding is to shock it for responding during a specific timed and signaled period. The animal is expected to maintain the response in the nonsignaled portions (ITI) of the training session. No sophisticated researcher would attempt to train a rat in a shuttlebox with this procedure because it would be very difficult, if not impossible, to distinguish the "learned inhibition" of response from the "habituated response" with a response incidence measure. The problem is exactly the obverse of the problem with goldfish in the active avoidance procedure. Thus, attempting to train goldfish with a passive or punishment procedure assumes, tacitly or otherwise, that the goldfish does not "habituate" its shuttle response.

Woodard and Bitterman (1973) trained goldfish to inhibit shuttling with a procedure in which the fish were shocked at the end of a timed and signaled period for responding during the period. Zerbolio and Wickstra (1978c) trained goldfish to inhibit responding using both the above procedure and also one in which the fish were shocked immediately for responding in the timed period. Since goldfish "learned" to inhibit responding, they are clearly sensitive to the punishment contingency. But Zerbolio and Wickstra (1978c) found equal or better response inhibition in a control that did not have the timed period signaled. Although the goldfish is sensitive to the punishment contingency it learns to inhibit shuttle responding in all periods of the session, not just during the timed period. Thus, although goldfish learn to inhibit response (passive avoidance) to avoid shock, they do not learn a stimulus-specific, or discriminated, passive avoidance response.

The last of these procedures is the successive presentation of two different signals, a procedure in which one signal ( $\mathrm{S}-$ ) marks a timed period that is paired with shock and the second signal $(\mathrm{S}+)$ marks a timed period that is never paired with shock. Woodard and Bitterman (1971) found that goldfish learned to respond in increasingly more $\mathrm{S}$ - signaled timed periods than they did in $\mathrm{S}+$ signaled timed periods. Zerbolio and Wickstra (1979b) found the same results, but found something else as well. If the $\mathrm{S}$ - signal is eliminated (turned off) so that the shock paired timed period is indistinguishable from the ITI, goldfish respond in increasingly more shock paired but unsignaled timed periods than they do in signaled $(\mathrm{S}+)$ and unpaired timed periods. The same discriminated pattern of response occurs both with or without the shock-paired timed period's being marked with a signal. A little thought will show that eliminating the $\mathrm{S}-$ signal from this successive presentation procedure turns it into an explicitly unpaired pseudoconditioning procedure, with the additional feature that responses are measured in two timed periods instead of just one. It is difficult to conclude that goldfish, trained with a successive presentation procedure, are learning a discriminated response pattern if the same kind of pattern occurs when one of the signals is eliminated. If the successive presentation procedure data (or explicitly unpaired pseudoconditioning data) represent any sort of learning at all, it is probably a learned inhibition to the unpaired CS, as suggested by Rescorla (1967). Thus, none of the goldfish shuttlebox studies referenced above seem to provide unequivocal evidence for the acquisition of a discriminated avoidance response in the shuttlebox. All the procedures and their results are rendered equivocal by the results of one or more control procedures. To circumvent the logical impasse created by the form of measurement characteristic of shuttlebox procedures, Zerbolio and Wickstra (1979a, 1980) used a Y-maze apparatus and a simultaneous presentation procedure in which the learning measure was not just the incidence of responding, but choice.

Using a Y-maze, Zerbolio and Wickstra (1979a, 1980) demonstrated that, with a trial-start (TS) signal, a shock-paired ( $\mathrm{S}-$ ) signal, and an unpaired signal $(\mathrm{S}+)$ presented simultaneously at the onset of a timed period, goldfish learned to swim from the TSsignaled alley they occupied at trial onset to the S+ signaled alternative alley within this timed period. Furthermore, when the two alternative alleys were signaled as being different from the TS alley but not different from each other, and when the signal differentiating shock pairing $(\mathrm{S}+/ \mathrm{S}-)$ was not presented until after the goldfish swam into one of the alternative alleys, they learned to leave the TS alley and swim to one of the alternative alleys. Then, using the response-contingent signaled information, goldfish either stayed in the initially chosen alley or left the initial choice and swam to the remaining alternative. The basis for this second response was the response-contingent signal. If the signal indicated no shock $(\mathrm{S}+)$, the goldfish stayed in the initially chosen alley for the remainder of the timed period. If the signal indicated shock pairing ( $\mathrm{S}-$ ), the goldfish left its initial choice and swam to the remaining alternative, 
where it received the $\mathrm{S}+$ signal. All responses occurred within the timed period of the trial prior to shock, and thus were avoidance responses. Although controls without the differential shock-pairing information continued to respond or swim from alley to alley, they did not learn to avoid above chance levels.

The reason the data collected in the Y-maze seem convincing is that the learning measure is not just the incidence of response, but of response and choice. To successfully avoid, the goldfish must learn not only to leave the TS alley, but also to choose the S+ alley. Simply swimming (leaving the TS alley) does not insure shock omission on more than half of the trials. Considering that Zerbolio and Wickstra's (1979a), 1980) criterion was visiting the $S+$ alley at least once on each trial, if the goldfish were to swim to each alley on each trial it could, of course, avoid as many shocks as the animal that responded selectively, but no evidence for superfluous responding was observed. Goldfish learned to avoid with a minimum number of responses, which implies a highly discriminated avoidance response.

Consider the sequence of events in the Y-maze procedure in which both alternative alleys are identical at trial onset and the signal differentiating shock pairing does not occur until after the goldfish makes its initial response.

The TS cue is presented at trial onset. The animal's initial response $\left(R_{1}\right)$ is to swim to one of the identically signaled alternative alleys. After this initial response, a second signal, either $\mathrm{S}+$ or $\mathrm{S}_{-}$, is presented. For the goldfish, these cues represent two distinct classes of events. If the $\mathbf{R}_{\mathbf{1}}$-contingent event is $S+$, the animal need not respond again to avoid shock. But, if the $R_{1}$-contingent event is $S-$, the animal must respond again $\left(R_{2}\right)$ by swimming from its initial choice to the remaining alternative. These classes of events are:

\section{Class 1, (TS) --- $\mathrm{R}_{1}---(\mathrm{S}+$ ) ---(no further response);}

$$
\begin{aligned}
& \text { Class 2, (TS) --- } R_{1}-\cdots(S-) \cdots R_{2} \cdots(S+) \cdots \\
& \text { (no further response). }
\end{aligned}
$$

Notice that both classes have in common the "TS -.. $\mathbf{R}_{\mathbf{1}}$..." events. Note also that all signal changes are the result of response contingencies. While it is true that, in the $\mathrm{Y}$-maze, these stimulus changes occur because the goldfish is transporting itself to different portions of the maze, the same essential stimulus changes could be produced in a shuttlebox with appropriate programming.

If, in a shuttlebox, trial start is indicated by a specific signal cue (TS), and if the goldfish's first response $\left(R_{1}\right)$ produces, at random, either a shock (US) paired $(\mathrm{S}-)$ cue or an unpaired $(\mathrm{S}+)$ cue, and if responses after $R_{1}$ produce alternation of the $S+$ and $S-$ cues, the same sequence of stimulus and response events that occurs in the Y-maze can occur in the shuttlebox. The US is administered, as in all avoidance paradigms, after a timed period following trial onset, but only if the goldfish fails to meet a specific response requirement. If the US is programmed to occur $10 \sec \left(T_{10}\right)$ after trial onset $\left(T_{0}\right)$, the US would be administered at $T_{10}$ if, at that time, the cue state of the apparatus was TS or $S-$, but would be omitted if the cue state was $\mathrm{S}+$. Thus, with this procedure, the learning measure is the proportion of trials on which the $S+$ cue prevails at $T_{10}$, not the number of trials with response. To meet this requirement, the goldfish must not only respond initially $\left(\mathbf{R}_{\mathbf{1}}\right)$, but must also use the responsecontingent cue information generated by its first response to decide whether or not to respond a second time. If, at $T_{10}$, the $S+$ cue prevails, the goldfish has necessarily met the response requirement.

Note that if the goldfish does not respond (no $R_{1}$ ), it cannot avoid, because the cue state would remain TS and shock would be presented at the end of the timed period. Randomly responding would not be effective either, since random response would produce, at best, the $S+$ cue state at $T_{10}$ on one-half of the trials. The only way the goldfish could avoid shock on better than one-half of the trials would be to learn to use the response-contingent cue change information and control the prevailing cue state of the box, so that the prevailing cue would be $S+$ at $T_{10}$.

If goldfish can learn to avoid in the shuttlebox when trained with the linear presentation procedure, the procedure becomes a logical alternative to the traditional two-stimulus successive presentation procedure, both in terms of the way the stimuli are programmed and, more importantly, in terms of the way learning is measured. Convincing evidence for the acquisition of a learned discriminated avoidance would be that goldfish learn to avoid on substantially more than one-half of the trials, and that they respond differently to the S+ and S- cues. Even more convincing would be evidence that goldfish reacquire the discriminated response when the shock pairing of specific cues is reversed.

The present experiment was designed to test the effectiveness of the linear presentation procedure with goldfish, both in an original learning phase and a reversal learning one. In addition, since goldfish possess color vision (Yeager, 1968) and since, although experimenters have used a variety of color cues (e.g., red/green, Woodard \& Bitterman, 1971; red/blue, Zerbolio \& Wickstra, 1979b), so far as is known, none has attempted a systematic comparison of different color cues in avoidance learning (as Muntz and Cronly-Dillon, 1966, did in a food reinforced procedure), the present experiment used all possible pairings of four different colors which constitutes six replications of the test of the linear presentation procedure. 


\section{METHOD}

\section{Subjects}

Sixty-four 5- to 6-cm-long goldfish, obtained from Ozark Fisheries and housed in individual $7.5 \times 11.5 \times 12.5 \mathrm{~cm}$ deep aquaria, served as subjects. Housing was well filtered, with both temperature $\left(21.1^{\circ} \mathrm{C}\right)$ and $\mathrm{pH}(7 \pm .1)$ held constant. The fish were fed daily.

\section{Apparatus}

Four identical $29.2 \times 11.4 \times 11.4 \mathrm{~cm}$ deep shuttle tanks, sim. ilar to those described by Horner, Longo, and Bitterman (1961), containing a $6.35-\mathrm{cm}$ center hurdle with $45^{\circ}$ sloping sides and a 9-cm-long flat top, were used. Water clearance over the hurdle was maintained at $2 \mathrm{~cm}$. Shuttling activity was monitored by photocells across the top of the hurdle at the ends of the top flat portion, $9 \mathrm{~cm}$ apart. Photocell light sources were $2.5-\mathrm{V}$ dc prefocused penlight bulbs, run at $1.5-\mathrm{V}$ ac to increase bulb life. Signal cue colors were provided by $7-\mathrm{W} 110-\mathrm{V}$ ac lamps. Colors used were red, yellow, green, and blue, with measured spectral peaks of $620 \pm 4 \mathrm{mu}$, $586 \pm 2 \mathrm{mu}, 517 \pm 2 \mathrm{mu}$, and $474 \pm 2 \mathrm{mu}$, respectively. Shock was delivered via $28 \times 10.2 \mathrm{~cm} \mathrm{22-ga}$ stainless steel plates which lined the interior sides of the tank. Shock was delivered at $7 \mathrm{~V} \mathrm{ac}$ $(.69 \mathrm{~V} / \mathrm{cm})$ in 200 -msec pulses. All events were programmed and recorded via appropriate circuitry. Running shuttle tanks were constantly aerated. Shuttleboxes and individual housing were cleaned daily with a suction cleaner, and approximately one-third of the water was replaced.

\section{Procedure}

The experiment was divided into two phases, an original learning (OL) phase and a reversal learning (REV) phase. Each phase lasted 10 days, for a total of 20 days in all. On each day, each goldfish was run 60 trials, with a variable interval of $60 \mathrm{sec}$ between trials.

Original learning. Forty-eight goldfish served as experimentals and 16 goldfish served as controls. In $\mathrm{OL}$, six experimental groups of 8 goldfish each were run, one group for each of the possible color combinations. Specifically, experimental groups had blue/ yellow, yellow/green, red/green, blue/red, red/yellow, or blue/ green color cues. In each group, one color was paired with shock $(\mathrm{S}-)$ and the other was never paired $(\mathrm{S}+)$. Furthermore, the color paired with shock was balanced within each experimental group; for example, half of the blue/yellow group had blue $S+/$ yellow $\mathrm{S}-$ and the other half yellow $\mathrm{S}+$ /blue $\mathrm{S}-$. For the controls, two groups of 8 goldfish were run with identical colors serving as $S+$ and $\mathrm{S}-$. Specifically, one control group had red/red, and the second blue/blue. The balancing-within-group procedure was followed for the controls. For measurement in the control groups, one of the identical stimuli was arbitrarily designated as the $\mathrm{S}+$. All animals were run for 10 days in the OL phase.

Reversal learning. In the reversal phase, haif of all groups had the shock pairing reversed and the remaining half continued, as in $\mathrm{OL}$, to serve as comparison controls. Specifically, each experimental and control group was balanced for color serving as $S+$. Half of each balanced subgroup had their $\mathrm{S}+$ and $\mathrm{S}$ - color cues reversed; for example, for the blue/yellow experimental group, which contained 8 goldfish, half were run with a blue $S+$ yellow $\mathrm{S}-$ and the remainder with a yellow $\mathrm{S}+$ /blue $\mathrm{S}-$ in $\mathrm{OL}$. Each of these balanced blue/yellow subgroups had 4 goldfish, and 2 of each had their $\mathrm{S}+/ \mathrm{S}-$ cues reversed and the remaining did not. The procedure was followed for all experimental and control groups to balance for all possible color and shock pairing possibilities. The following general procedure was used in both phases of the experiment.

General procedure. For the six experimental groups, six colored bulbs, three of each color, served to provide all illumination cues. The intertrial-interval (ITI) cue was the illumination of all six bulbs. The trial start (TS) cue was the extinguishing of all six bulbs, rendering the shuttlebox dark. The $\mathrm{S}+$ and $\mathrm{S}-$ cues were pro- vided by sets of three bulbs, one on one end of the tank and two on the other end, arranged so that, at one end, one color was flanked by two bulbs of the other color (e.g., blue/yellow/blue for one end and yellow/blue/yellow for the other). Balancing of color and bulb position was necessary because earlier pilot work in our lab had found that with a compound ITI cue (S+ and $\mathbf{S}-$ ), some goldfish learned to avoid using bulb position (side vs. center) rather than color. The control groups differed only in that all six bulbs were the same color.

A trial was a 11.2-sec period. The first $10 \mathrm{sec}$ were shock-free, and were followed by two 200 -msec US pulses, with an 800 -msec intershock interval (ISI). By proper response, goldfish could prevent either one or both of the shock pulses. US presentation was entirely dependent on the response-contingent cue state of the shuttlebox at the programmed times of delivery (first pulse at $T_{10}$ and a second at $\left.T_{11}\right)$. The exception to this was if the initial response $\left(R_{1}\right)$ did not occur until after the first US pulse.

The trial start $\left(T_{0}\right)$ to first US $\left(T_{10}\right)$ interval. If no response occurred in the $T_{0}$-to- $T_{10}$ interval, the shuttlebox cue state remained TS (dark) and the first US pulse was delivered as programmed. If $R_{1}$ occurred in the $T_{0}$-to- $T_{10}$ interval, $R_{1}$ produced, at random (Gellerman series), either the $S+$ or $S$ - cue. Further responses at any time during the remainder of the trial (from $R_{1}$ to $T_{112}$ ) produced the alternation of the $S+$ and $S$ - cues (e.g., from $S+$ to $S-$ or from $S-$ to $S+$ ). Once eliminated by $R_{1}$ in the $T_{0}-t_{0}-T_{10}$ period, the TS cue did not recur until the onset of the next trial. If, at $T_{10}$, the prevailing cue was $S+$, the first US pulse was omitted. If the prevailing cue was $S_{-}$, the first US pulse was delivered as programmed.

The $T_{10}$ to $T_{11.2}$ interval. Omission of the first US pulse did not guarantee omission of the second. If the animal shuttled during the 800 -msec ISI interval between programmed US pulses and changed the prevailing cue state from $S+$ to $S-$ so that $S$ - prevailed at $T_{11}$, the second US pulse was delivered as programmed. Conversely, if the first US pulse was delivered at $T_{10}$ because the prevailing cue state was $S-$ and the goldfish shuttled during the ISI, the prevailing cue state changed to $\mathrm{S}+$ and the second US pulse was omitted. Thus, for the goldfish to avoid both US pulses, the animal would have to respond during the $T_{0}$-to- $T_{10}$ intervaland, via its response, establish the $S+$ cue so that at $T_{10}$ the prevailing cue was $S_{+}$- and, further, to maintain the $S+$ cue by not responding again during the entire US delivery period.

A remaining special case must be detailed. If the goldfish did not respond during the $T_{0}$-to- $T_{10}$ interval, the TS or trial start cue prevailed at $T_{10}$ and the first US pulse was delivered as programmed. If a response on any given trial does not occur until after the first US pulse, one can argue that the response is shock-elicited, and therefore different from responses occurring earlier in the trial period before shock. Initial responses occurring after the first shock pulse were treated as special cases in that they did not produce illumination cue change-[that is, the TS cue (dark) remained for the rest of the trial regardless of the number of responses]. However, occurrence of the second US pulse was still a responsecontingent event. If the $R_{1}$ response occurring prior to $T_{10}$ produced (at random) the $S+$ cue, the second US pulse was omitted, but if the $R_{1}$ response occurring prior to $T_{10}$ produced $S-$, the second US pulse was delivered as programmed. Thus, the goldfish received the second US pulse on half of the trials when its first response occurred after $T_{10}$.

Response measures. The main focus of the experiment was to examine avoidance behavior. Thus, only the responses occurring in the $T_{0}-t_{0}-T_{10}$ timed period were measured. These were divided into two classes of response, differing in the cue presented contingent on the first $\left(R_{1}\right)$ response. The two classes were:

Class 1, (TS) -.- $\mathbf{R}_{1} \ldots$ - (S+) - - (measurement of response frequency);

Class 2, (TS) - - $\mathrm{R}_{1} \ldots$ (S-) - - (measurement of response frequency). 
In addition to responding initially to the TS cue, one would expect that goldfish would learn not to respond again if their initial response yielded S+ (Class 1) but to respond a second time if their initial response yielded the $\mathrm{S}-$ cue (Class 2). The frequency of responses in each of these two classes was recorded. Also, the number of trials without shock (avoidances) was recorded. The shock could be omitted on only those trials in which the $S+$ cue prevailed for the entire shock delivery period, or $T_{10}$ to $T_{11.2}$. Animals could respond several times in the timed interval prior to shock and still meet the cue state requirement at $T_{10}$. The frequency of response in Class 1 and Class 2 and the trials with avoidance were designed to measure two different aspects of avoidance performance. In addition, with the use of a compound ITI cue composed of both the S+ and S- light elements, it was possible that the goldfish would learn to spend the ITI period on the end of the shuttlebox with two $S+$ and one $S-$ lamps rather than two $S-$ and one S+ lamp. If so, the animal would be starting each trial from the same end. To determine this, the specific end the goldfish occupied at the beginning of every trial was recorded. Finally, the number of shuttles occurring during the ITI for each animal was recorded.

\section{RESULTS}

The data from the OL and REV phases were separated for analysis. Each phase was analyzed, using repeated measures ANOVA procedures, and when necessary to clarify the results, using additional partitions. All experimental groups were pooled and compared with the combined control group unless otherwise indicated.

\section{Phase 1: Original Learning}

The major results for the OL phase are shown in Figure 1. The top portion shows the daily percentage of trials in which the goldfish did not receive shock (avoidance) and the appropriate comparison baseline, the chance avoidance level. Recall that, in the linear presentation procedure, like a simultaneous presentation procedure, the randomly responding animal will produce the $S+$ cue condition, by chance, on $50 \%$ of the trials. For the animal to avoid on more than $50 \%$ of its response trials, it must use the responsecontingent cue information and adjust the cue state by responding accordingly. For comparison, across days, the top of Figure 1 shows the mean observed percentage avoidance and $50 \%$ chance baseline both for each of the experimental replications and for the combined controls. As the top of Figure 1 shows, with training, all groups show an increase in avoidances and in trials with response, but only the experimental groups show an increase in avoidances above the $50 \%$ random chance baseline. Two facets of the top of Figure 1 are striking. First, all of the

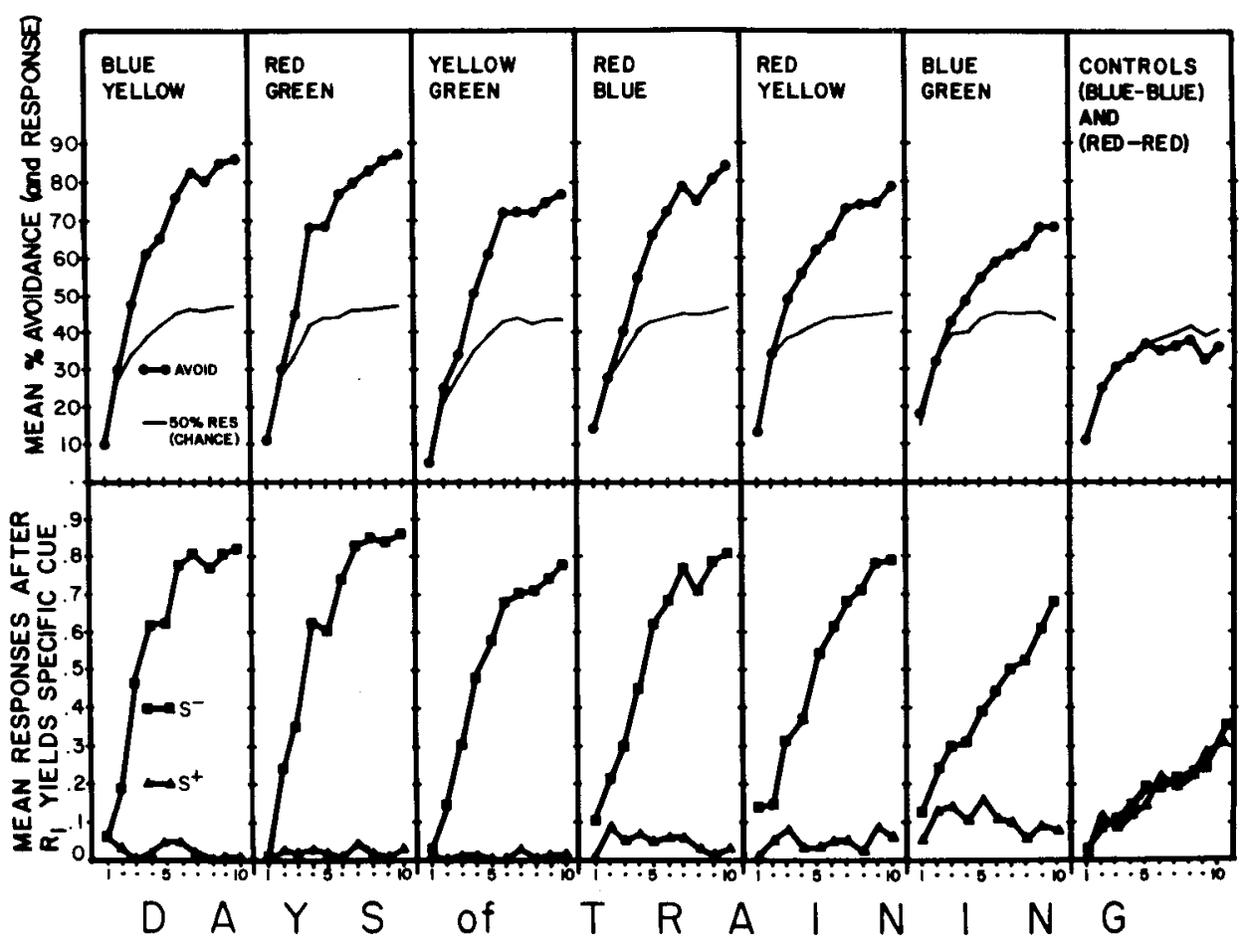

Figure 1. Two distinctly different measures of learning are shown in the figure. In the top panels, the dally mean percent observed avoidance levels and daily mean percent chance avoidance (50\% of the trials with response) levels for all experimental and the combined control groups are shown. In the bottom panels, the daily mean responses to the specific cues generated by the initial response, $\mathrm{S}+$ and $\mathrm{S}-$, are shown for all experimental groups and for the combined controls, indicating the highly discriminated and stimulus-specific character of the avoidance response. 
experimental groups, which are provided with adequate shock-pairing information, learn to avoid well above the chance avoidance expectancy, and second, the controls, despite the fact that they never receive sufficient information to determine shock pairing, respond on increasing proportions of trials with training. These results were confirmed by an analysis of the number of trials with response and the number of trials with avoidance.

On the trials with response, the number of responses of all groups, experimental and control, increased with training $[\mathrm{F}(9,558)=308.99, \mathrm{p}<.01]$, but the experimental goldfish responded on significantly more trials than the controls $[F(1,62)=9.08$, $\mathrm{p}<.01]$. A training $\times$ group interaction was also observed $[F(9,558)=3.10, p<.01]$.

On the trials with avoidance measure, all groups showed an increase in avoidances with training, as would be expected $[F(9,558)=292.18, p<.01]$, but the experimental group avoided on substantially more trials than the controls $[F(1,62)=104.16$, $\mathrm{p}<.01]$. A training $\times$ group interaction was also observed, as expected $[F(9,558)=40.03, p<.01]$.

The second learning measure was designed to determine the specificity of the discriminated response. Recall that when the goldfish responds to the TS cue, its response will yield, randomly, either the $S+$ or the $\mathrm{S}-$ cue, which represent, for the goldfish, two distinctly different classes of events. If the first response $\left(R_{1}\right)$ yields $S_{+}$, no further response is necessary to avoid on that trial. If, however, the first response $\left(R_{1}\right)$ yields the $S-$ cue, the goldfish would have to respond again $\left(R_{2}\right)$ to produce a cue change to $S+$ in order to avoid shock. If the goldfish learned to use the $\mathrm{S}+/ \mathrm{S}-$ cue information most efficiently, it would be expected to respond a second time $\left(R_{2}\right)$ only when its initial response yielded the $S$ - cue, but not to respond if the initial response yielded the $S+$ cue. To determine if the goldfish learns to use the cue information efficiently, the mean numbers of responses in these two classes were recorded and appear in the bottom of Figure 1.It is clear that all experimental goldfish learn to respond quite efficiently. They do not respond following $R_{1}$ when $R_{1}$ yields the $S+$ cue, but when $R_{1}$ yields the $S$ - cue, the mean number of responses, over training, rapidly approaches 1.00 , precisely the level of the most efficient response pattern. The pattern of response is clearly present in all the experimental groups, whereas, in contrast, the controls, which do not receive differential shock pairing information, learn to respond indiscriminately no matter what cue occurs as a result of their first $\left(R_{1}\right)$ response. The analysis of the $S+$ vs. S- responses over days of training confirms this impression.

The initial analysis, along with the finding of a significant difference between the experimental and control animals $[F(1,62)=23.72, p<.01]$, found sig- nificant values for all other main effects and most interactions. To clarify this analysis, the data were partitioned into the experimental and control segments and each was tested separately. For the control group, an increase in responses to cues following $R_{1}$ was found $[F(9,558)=17.38, p<.01]$, but responses to the $S+$ and $S-$ cues did not differ $(F<1)$. For the experimentals, an increase in second responses with training occurred $[F(9,558)=94.83$, $\mathrm{p}<.011$, but it was quite cue specific. Experimental goldfish responded increasingly more often to the $\mathrm{S}-$ than to the $S+$ cue $[F(1,62)=733.17, p<.01]$, and there was, as expected, a $S+$ vs. $S-x$ training interaction $[F(9,558)=132.19, p<.01]$. Clearly, the experimentals learn a highly efficient and cue-specific discriminated response.

Additionally, responses during the ITI and the starting position (shuttle tank end occupied at the start of the trial) were analyzed. Experimentals and controls did not differ in ITI responses $(F<1)$, but all showed a significant decrease in ITI response, from an average of 37.7 on Day 1 to 17.0 on Day 10 $[\mathrm{F}(9,558)=12.74, \mathrm{p}<.01]$. No training $\times$ group interaction was found $(F<1)$. Animals also never departed from a random expectancy in starting position, indicating that the compound ITI stimulus ( $\mathrm{S}+$ plus $\mathrm{S}_{-}$) did not produce differential effects during the ITI.

\section{Phase 2: Reversal Learning}

The major results of the REV phase are shown in Figure 2. As before, Figure 2 is divided into a top and bottom section, representing the two different measures of learning as detailed for Figure 1.

The top of Figure 2 shows both the percentage chance avoidance level and the percentage observed avoidance level for both the experimental and control groups that had shock pairing reversed (OL-REV) and for those that continued as in original learning (OL-OL). It is clear that those goldfish with cues maintained as in original learning (OL-OL) continued to avoid well above chance avoidance levels. It is also clear that the reversed goldfish (OL-REV) dropped below chance levels on the first day of reversal, but, with training, learned the reversed cue shock pairing and reacquired the discriminated avoidance response with the reversed cues. The reversed goldfish learned to avoid at levels comparable to the OL-OL animals after several days of training. For the controls, their performance remained at or below chance for the entire second phase, as expected. Besides the clear reversal learning obvious in Figure 2, note that the chance avoidance levels, or $50 \%$ of the trials with a response, are remarkably stable for all groups throughout the second phase of training. This is also true of the control groups, even though they never are provided with sufficient information to de- 


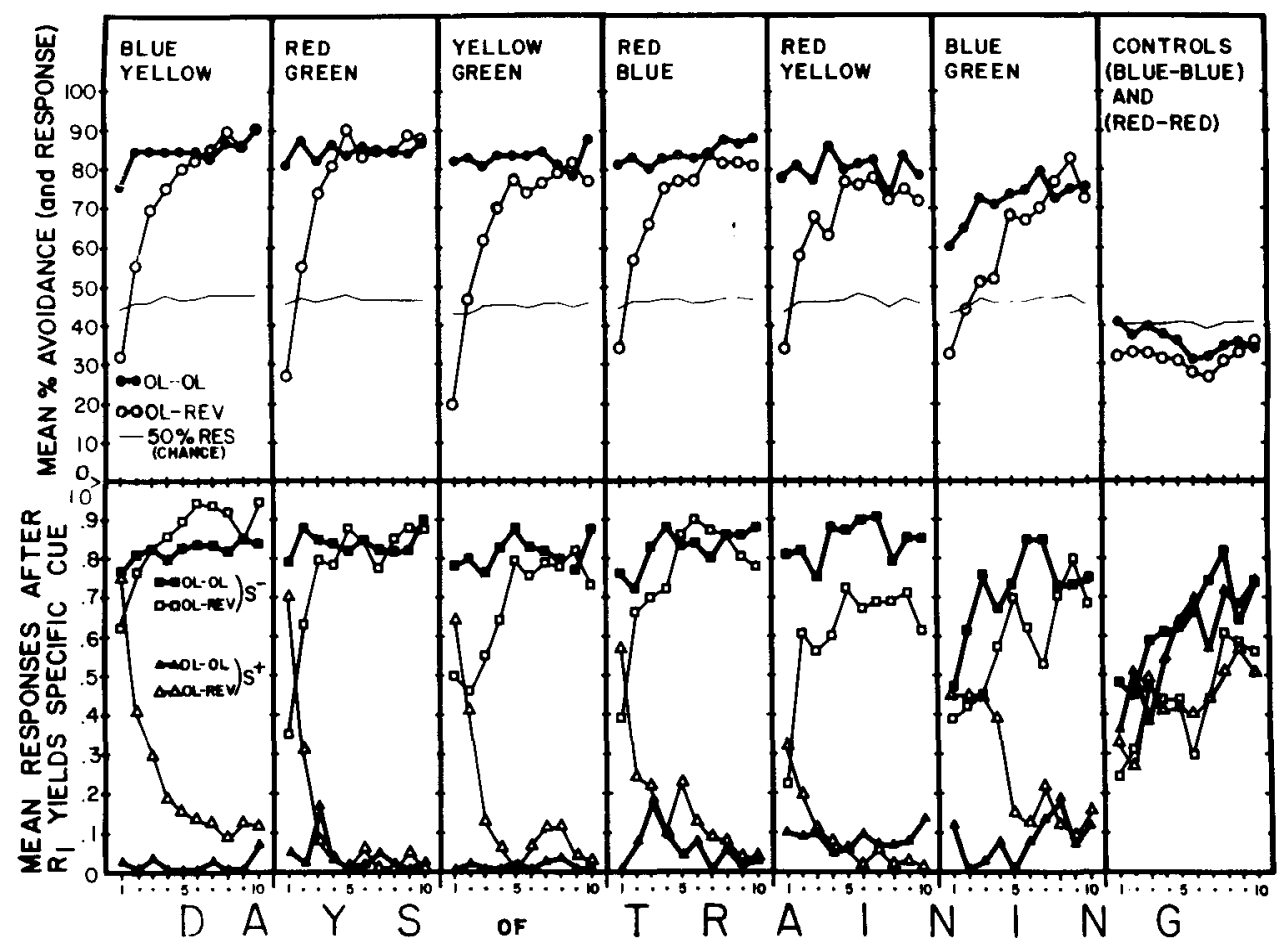

Figure 2. Two distinctly different measures of learning are shown in the figure. In the top panels, the daily mean percent observed avoidance levels and daily mean chance avoidance levels for all experimental groups, both with the original learning shock pairing maintained (OL-OL) and with the original learning shock pairing reversed (OL-REV), and for the combined controls are shown. In the bottom panels, the dally mean responses to the cues generated by the initial response, $S+$ and $S-$, are shown for all OL-OL. and OL-REV experimental groups, and for the combined controls, indicating the highly stimulus-specific character of the avoidance response both for the OL-OL experimental groups and, by the end of training, for the OL-REV experimentals as well.

termine shock pairing. All the results shown in the top of Figure 2 were confirmed by analysis. For the trials with response measure, reversed and original learning animals, regardless of group, did not differ $[F(1,60)=2.64]$, nor did reversal interact with training or experimental vs. control grouping. But the experimental groups responded on significantly more trials than the controls $[\mathrm{F}(1,60)=50.99, \mathrm{p}<.01]$. With training, all groups showed a slight increase in trials with response $[F(9,540)=4.64, p<.01]$. No other effects or interactions were observed.

For the avoidance measure, the initial analysis found a huge difference between experimental and controls $[F(1,60)=272.84, p<.01]$, and significant values for all other main and interactive effects. To clarify the result, the analysis was partitioned into the experimental and control sections and each section was analyzed separately. For the control groups, no differences in avoidance due to reversal $[\mathrm{F}(1,60)=$ $1.23]$, due to training $[F(9,540)=1.46]$, or their interaction $(F<1)$ were found. The analysis of the experimental groups found significant differences due to the reversal of cues $[F(1,60)=22.20, p<.01]$, due to training $[F(9,540)=57.31, \mathrm{p}<.01]$, and a reversal $\times$ training interaction $[F(9,540)=35.28, \mathrm{p}<.01]$, exactly as would be expected from Figure 2 .

As with the original learning phase, a second learning measure to determine the efficiency of the discriminated response was also taken and the results appear in the bottom portion of Figure 2. As can be seen there, all OL-OL experimental groups maintained their discriminated performance by continuing to respond a second time only when their initial response $\left(R_{1}\right)$ yielded an $S$ - cue. If their initial response $\left(R_{1}\right)$ yielded $S+$, these OL-OL goldfish did not respond again. For the experimentals with their OL shock pairing reversed (OL-REV), they learned, with training, to reverse their responses and, by the end of the second phase, were responding quite efficiently to the reversed cues. For the controls, there was simply an increase in second response with no sign of discrimination, which is exactly what was expected. These results were confirmed in detail by the analysis which compared $\mathrm{S}+$ and $\mathrm{S}-$ responses, for OL-OL and OL-REV halves of each group, over the 10 days of training of the second phase. In the initial analysis, although the experimental and control groups, did not differ as a main effect $[F(1,60)=$ 
2.48], a very large group $\times S+/ S-$ interaction $[F(1,60)$ $=228.39, \mathrm{p}<.01]$ was observed, as were many other interactions. To clarify the results, the analysis was partitioned into experimental and control group sections, and each section was analyzed separately.

For the control groups, the only significant effect observed was an increase in the frequency of second responses $[F(9,540)=10.61, p<.01]$. No other significant effects were observed.

For the experimental groups, neither reversal of cues nor training by itself was significant (both Fs < 1), but significant values were found for differences in response to cues $S+/ S-[F(1,60)=963.21, p<.01]$, and for the $S+/ S-\times O L / R E V \quad[F(1,60)=27.54$, $\mathrm{p}<.01]$, the $\mathrm{S}+/ \mathrm{S}-\times$ training $[\mathrm{F}(9,540)=40.15$, $\mathrm{p}<.01]$, and the $\mathrm{S}+/ \mathrm{S}-\times \mathrm{OL} / \mathrm{REV}$ by training $[F(9,540)=24.45, p<.01]$ interactions. This is exactly what would be expected if, to determine the response to a specific cue, you would need to know which cue, if it had been reversed, and when in the reversal training procedure the cue occurred. This pattern conforms exactly to the data shown for the experimentals in the bottom portion of Figure 2. Thus, in the reversal phase of the experiment, goldfish learned to follow the reversed cues, or if the cueshock pairing was not reversed, to maintain response to the original cues, but, in either case, by the end of the reversal phase, all experimental groups were avoiding in a highly efficient and discriminated manner.

As in the first phase, the ITI responses were compared, and, in the initial analysis, both training $[F(9,540)=4.36, p<.01]$ and training $\times$ group ef fects $[F(9,540)=2.47, p<.01]$ were found. A partition revealed that there were no differences in ITI response for the controls across the second phase (all Fs $<1$ ). For the experimentals, the reversed goldfish showed more ITI responses on the first day of reversal than did the other animals (28.2 vs. 17.4$)$ but then settled down to a level comparable to that for the control and experimental animals thereafter (17.1).

As in the first phase, the starting position of all goldfish at the beginning of trials was recorded, but no end preference was observed.

\section{Color Analysis}

The six different experimental groups represent all possible pairs of four colors, red, blue, green, and yellow. The data for the pooled experimental groups clearly show that all groups, regardless of the specific color combination used, learn to discriminatively avoid when trained with the linear presentation procedure. However, perusal of the figures suggests that the blue/green color combination is more difficult to learn than the other color combinations. This would be consistent with the findings of Muntz and CronlyDillon (1966). However, Muntz and Cronly-Dillon reported that the blue/green effect was dependent on which color was paired with the positive reinforcer, the discrimination being learned quicker when the blue cue was positive than when the green cue was positive. In the present experiment, the balanced halves of each experimental and control group were compared to determine if pairing one cue with shock (e.g., blue $\mathrm{S}+$ /green $\mathrm{S}-$ ) produced better performance than the other pairing (e.g., green $S+/$ blue $\mathrm{S}-$ ). In no case was a significant difference between balanced halves of a specific color combination observed. Additional analyses compared all different color experimentals (and controls) to determine if the blue/green group was, indeed, statistically different from the other groups. In Phase 1, the six different color experimental groups did not differ in trials with response $[F(5,56)=1.03]$ or in avoidances $[F(5,56)=$ 2.23). All groups also showed the $\mathrm{S}+/ \mathrm{S}-$ discrimination with the lowest significant value for the blue/ green pairings, if $F(1,504)=76.24, p<.01$, can be called a "low value." All experimental groups were also equivalent on ITI responses. Similar analyses were conducted for the reversal phase. Again, the different color experimental groups did not differ in trials with response $(F<1)$. Differences between different color experimentals were observed on the avoidance measure $[F(5,48)=2.66, p<.05]$ but were not robust enough to locate with individual test procedures. No reliable effects were found that could be traced to the blue/green group in the $\mathrm{S}+/ \mathrm{S}-$ measure. Thus, although the blue/green color group appears to perform less well than the remaining color experimentals, statistical evidence supporting this apparent difference was not clearly found in these data.

What is clear from these data is that goldfish are trichromatic and can use a variety of color cues efficiently, which aids the experimenter in choosing cues to use as discriminanda.

\section{DISCUSSION}

These data clearly show that, when trained with the linear presentation procedure adapted from the Y-maze, goldfish, in the shuttlebox, learn a highly efficient and discriminated avoidance response, and further, when the specific shock pairing for different cues is reversed, they learn the reversal. Like the simultaneous-presentation $\mathrm{Y}$-maze procedure, the linear presentation shuttlebox procedure seems to be immune to attempts to account for the results with a random response increase explanation, because any sort of random increase could produce, at best, avoidance on a maximum of half of the trials with response. All experimental groups show avoidance levels well above the random chance $(50 \%$ of the trials with response) avoidance baseline. As in the Ymaze, with stimuli presented simultaneously, gold- 
fish trained in the shuttlebox with the linear presentation procedure can serve as their own activity or random response control. The specificity of the avoidance response goldfish learn is clearly shown in the bottom portions of Figures 1 and 2 . It is readily apparent that, if the first response $\left(R_{1}\right)$ yields the shockpaired $S-$ cue, they learn to respond a second time to change the cue state to $S+$, but if $R_{1}$ yields the unpaired cue $(\mathrm{S}+)$, they stay put. It is indeed tempting to conclude that these data represent the acquisition of a discriminated avoidance response via an instrumental process, as do similar data collected in the Y-maze (Zerbolio \& Wickstra, 1979a, 1980), but there may be another explanation.

To account for their results in both one- and twosignal avoidance procedures, Woodard and Bitterman $(1971,1973)$ hypothesized that goldfish learned, via a classical process, to specifically respond during shock-paired periods and not to respond during unpaired cue periods. If this explanation is valid for the present experiment, there should be a high correlation between responses in the two shock paired periods (S - and TS) and a comparable high correlation between the responses in the unpaired cue periods ( $\mathrm{S}+$ and ITI). To test this interpretation, correlations between mean responses for the TS and $S$ - cue periods, for all experimentals for the first 10 days, for the OL-OL experimental groups for the second 10 days (Days 11 through 20), and for the OL-OL experimentals over all 20 days of training were calculated and found to be $r(8)=.92, r(8)=.92$, and $r(18)$ $=.92$, respectively (all ps $<.01$ ). Comparable correlations of the mean $\mathrm{S}+$ and mean ITI responses over days of training were $r(8)=-.47, r(8)=.12$, and $\mathrm{r}(18)=-.02$, respectively (all ps $>.05$ ). Thus, while the responses in the shock-paired cue periods are highly correlated, the responses in the unpaired or safe periods are not related, which militates against the classical activation hypothesis.

More traditional avoidance learning positions, all of which assume an instrumental process, would have no trouble with these data. Two-factor theory would simply assume that, via shock pairing, two cues, S- and TS, are fear cues and the goldfish simply continues to shuttle until its response produces a cue that does not elicit fear (and its accompanying escape response). More current theories, such as Bolles (1978) or Seligman and Johnston (1973), would assume that the $S+$ cue indicates saîety or a low probability of shock. Any one of these positions can easily account for these results. But the nature of the discrimination seen here deserves further note.

In avoidance learning, the meaning of "discriminated avoidance" has come to imply that the animal responds to a specific cue that is presented during a timed and signaled period before shock. This meaning is different from that of "discrimination learn- ing" as it is used in the "positive or food reinforcement" literature. With food reinforcement, the term typically means that the animal learns to respond differentially to two different cues. With the standard laboratory behavioral preparations, discriminated avoidance in the positive reinforcement sense of the term, has been exceedingly difficult to produce, as the paucity of such work in the avoidance literature indicates. Goldfish, both in the Y-maze and, with these data, in the shuttlebox, seem to show precisely such a response, that is, learning to respond differentially to two differently reinforced cues. It is possible that goldfish may open up the entire area of discrimination learning to the avoidance researcher, an area that has to date been the exclusive province of researchers using positive reinforcement procedures. The behavioral characteristic of goldfish that may make this possible is the finding that, in procedures without shock, goldfish do not show the expected habituation of responding, but, with "training," increase the proportion of timed periods with a response. Retrospectively, this result, reported initially by Steiner (1971) and observed later by Zerbolio and Wickstra (1978a, 1978b), forces the following question: What is "habituation for the goldfish"? It seems that the behavioral manifestation of "habituation" should be operationally defined as the result of the "habituation measurement procedure." While it is true that we expect animals to stop responding in the shuttlebox during "habituation procedures," that is only because we are used to seeing this result in the bulk of the animals tested. Rats, cats, dogs, and mice all habituate, that is, stop responding, but is habituation necessarily the cessation of response? Because the goldfish's behavior does not meet our preconceptions of a habituated response, does that mean the goldfish is wrong? Perhaps the problem isn't with the goldfish, but in our preconceptions of what constitutes a "habituated response." We may expect a cessation of response, but nobody told the goldfish. If we make the simple assumption that the habituated response in goldfish is an increase in responding, then almost all of the problems in avoidance learning with goldfish disappear. Of course, like trying to teach a rat a passive avoidance response in the shuttlebox, we have to devise a learning measure that allows us to discriminate the "habituated response" from the "learned response." The Y-maze procedure and the linear presentation procedure in the shuttlebox may be just the appropriate measures because both require not only that the goldfish "increase response" (habituate), but that it learn a choice response as well. It is the choice response-response and attendant choice in the Y-maze with $\mathrm{S}+$ and $\mathrm{S}-$ presented simultaneously at trial onset (Zerbolio \& Wickstra, 1979a, 1980), response and subsequent choice in the Y-maze with alternative alleys iden- 
tically cued at trial onset and shock pairing information contingent on an initial response (Zerbolio \& Wickstra, 1980), and response and subsequent choice in the shuttlebox with the linear presentation procedure-that allows the experimenter to separate the "learning response" from the "habituated response."

The identical color controls in the present experiment give credence to this point of view. Despite the fact that they cannot tell when shock will occur, they not only "learn" to increase responding in the first phase, but maintain it in the entire second phase even though they receive shock on $66 \%$ of the trials. If this is assumed to represent their normal pattern of "habituation," there is nothing unusual about their behavior at all. It is perfectly consistent with all the other work reported in this paper. The importance of this pattern of habituation for avoidance learning research is that the goldfish represents an animal that does not "freeze" in shock situations. If the goldfish does not "freeze," it offers the experimenter the opportunity to differentially reinforce its behavior with shock omission, and thus to measure the effect of the differential reinforcement. The typical laboratory animal, that is, the rat, will learn to freeze if its SSDRs are not reinforced fairly quickly by shock omission (Bolles, 1970). Once the animal learns to "freeze" in the timed and signaled period prior to shock, the opportunity to differentially reinforce with shock omission is lost, and the capability of the animal to learn a discriminated avoidance response comparable to what a goldfish can learn is moot. Goldfish continue to respond, even when shocked, as long as they are not expressly punished for responding, and thus provide the experimenter with the opportunity to differentially reinforce with shock omission.

The linear presentation procedure does have some logical advantages over the $\mathrm{Y}$-maze. For instance, in the $\mathrm{Y}$-maze, with $\mathrm{S}+$ and $\mathrm{S}-$ presented simultaneously, the experimenter cannot be sure the animal is equally exposed to both the $S+$ and $S$ - cues because the animal could make its choice by attending to either cue by itself. In the linear presentation procedure, the animal is forced to attend to each cue on a random half of all trials with an initial response. Another advantage to the linear presentation procedure is that it has an automatic correction for random multiple response. For instance, if the goldfish responds during the $S+$ cue, the cue changes. The animal is therefore potentially punished for a superfluous random response.

The last advantage the linear presentation procedure may have is that it still uses the venerable shuttlebox. There are many more labs around that have shuttleboxes than have Y-mazes. It is hoped that these data, which show the acquisition of a discriminated avoidance response in goldfish, discriminated in the positive reinforcement sense, will encourage researchers to do further work.

In sum, then, it seems that goldfish can and do learn a highly efficient discriminated avoidance response in the shuttlebox. Not only do they learn an original discriminated avoidance response, but when the cue-shock pairing relationship is reversed, they also learn the reversal. All these data point to an instrumental process. However, as a word of warning, these data may be restricted to the kind of shuttlebox described in the apparatus section, a shuttlebox similar to that described by Horner, Longo, and Bitterman (1961). This shuttlebox has a hurdle with 45-deg sloping ramps and a top flat which may encourage activity in this animal. Other types of "shuttleboxes," such as that used by Scobie and Fallon (1974), have a vertical barrier hurdle instead of the sloping ramp hurdle. It is not presently known if these conclusions apply to the barrier hurdle type of shuttlebox. Lastly, avoidance learning in goldfish is controversial. Hopefully the present data and analysis will serve to reduce some of the controversy, but it is quite possible, given the past history of this animal in this apparatus, that the present data may end up posing more questions than they answer.

\section{REFERENCES}

Bolles, R. C. Species-specific defense reaction and avoidance learning. Psychological Review, 1970, 77, 32-48.

Bolles, R. C. The role of stimulus learning in defensive behavior. In S. H. Hulse, H. Fowler, \& W. K. Honig (Eds.), Cognitive processes in animal behavior. Hillsdale, N.J: Erlbaum, 1978.

Horner, J. L., Long, N., \& Bitterman, M. E. A shuttlebox for fish and a control circuit of general applicability. American Journal of Psychology, 1961, 74, 114-120.

Muntz, W. R. A., \& Cronly-Dillon, J. R. Color discrimination in goldfish. A nimal Behaviour, 1966, 14, 351-355.

Rescorla, R. S. Pavlovian conditioning and its proper control procedures. Psychological Review, 1967, 74, 72-80.

Scobie, S. R., \& Fallon, D. Operant and Pavlovian control of a defensive shuttle response in goldfish (Carassius auratus). Journal of Comparative and Physiological Psychology, 1974, 86, 858-866.

Seligman, M. E. P., \& Johnston, J. C. A cognitive theory of avoidance learning. In F. J. McGuigan \& D. B. Lumsden (Eds.), Contemporary approaches to conditioning and learning. Washington, D.C: Winston, 1973.

StE INER, G. Stimulus control of avoidance learning in fish. Journal of Comparative and Physiological Psychology, 1971, 74, 52-58.

Woodard, W. T., \& Bitterman, M. E. Classical conditioning of goldfish in the shuttlebox. Behavior Research Methods \& Instrumentation, 1971, 3, 193-194.

Woodard, W. T., \& Bitterman, M. E. Pavlovian analysis of avoidance conditioning in the goldfish (Carassius auratus). Journal of Comparative and Physiological Psychology, 1973, 82, 123-129.

YAGER, D. Behavioral analysis of color sensitivities in goldfish. In D. Ingle (Ed.), The central nervous system and fish behavior. Chicago: University of Chicago Press, 1968.

Zerbolio, D. J., JR., \& Wickstra, L. L. Does elimination of a negative phototaxis eliminate CAR acquisition in goldfish? Bulletin of the Psychonomic Society, 1978, 11, 213-216. (a) 
Zerbolio, D. J., JR., \& Wickstra, L. L. Goldfish avoidance acquisition: Is the process classical, instrumental, or a phototaxis? Bulletin of the Psychonomic Society, 1978, 11, 321-323. (b)

Zerbolio, D. J., JR., \& Wickstra, L. L. Passive avoidance in goldfish: Lack of evidence for stimulus specificity. Bulletin of the Psychonomic Society, 1978, 12, 15-17. (c)

Zerbolio, D. J., JR., \& Wickstra, L. L. Discriminated response patterning in goldfish with successive presentation and explicitly unpaired pseudoconditioning procedures. Bulletin of the Psychonomic Society, 1979, 13, 307-310. (a)
Zerbolio, D. J., JR., \& WICKstra, L. L. Instrumentally based conditioned avoidance response acquisition in goldfish in a simultaneous presentation task. Bulletin of the Psychonomic Society, 1979, 13, 311-313. (b)

Zerbolio, D. J., JR., \& Wickstra, L. L. Instrumental avoidance acquisition by goldfish in a Y-maze using explicit and responsecontingent cues. Animal Learning \& Behavior, 1980, 8, 304-310.

(Manuscript received January 30, 1981; revision accepted for publication April 16, 1981.) 